\title{
A PLANAR ROTOR MODEL FOR CHOLESTANE SPIN LABEL MOTION IN PHOSPHOLIPID MULTIBILAYERS WITH HIGH ORDER
}

\author{
A.J. DAMMERS \\ Centre for Submicron Technology, Delft University of Technology, P.O.Box 5046, 2600 GA Delft, The Netherlands ${ }^{I}$ \\ and Department of Molecular Biophysics, Physics Laboratory, University of Utrecht, \\ Princetonplein 5, 3584 CC Utrecht, The Netherlands

\section{Y.K. LEVINE} \\ Department of Molecular Biophysics, Physics Laboratory, University of Utrecht, \\ Princetonplein 5, 3584 CC Utrecht, The Netherlands

\section{K. BALASUBRAMANIAN and A.H. BETH} \\ Department of Molecular Physiology and Biophysics, Vanderbilt University School of Medicine, Nashville, TN 37232, USA
}

Received 7 September 1987 ; in final form 25 July 1988

\begin{abstract}
An EPR study of a macroscopically oriented multibilayer system of DMPC/cholesterol $(2: 1)$, order parameter $S \approx 1$, is presented. The conventional cholestane spin label ${ }^{14} \mathrm{~N}-\mathrm{CSL}$ as well as its isotopically substituted analogue ${ }^{15} \mathrm{~N}-\mathrm{CSL}$ were used in the experiments. The temperature range studied, -18 to $18^{\circ} \mathrm{C}$, covered the rigid-limit and the slow-motion regime. The experimental spectra were simulated by a numerical solution of the stochastic Liouville equation using Padé approximant techniques. As the ordering of the CSL molecules in this bilayer system is virtually indistinguishable from perfect alignment of the long molecular axes along the director (the normal to the multibilayer plane) the planar rotor model was used to describe the reorientational motions. Thus the molecules were taken to possess only a single rotational degree of freedom about their long axes. The analysis of the slow-motion spectra reveals that the CSI. molecules undergo a random rotational jump motion rather than small-step Brownian diffusion. The use of both the ${ }^{14} \mathrm{~N}-\mathrm{CSL}$ and ${ }^{15} \mathrm{~N}$-CSL greatly improves the reliability of the spectral simulations. Furthermore the use of the ${ }^{15} \mathrm{~N}$-CSL molecules affords a unique mapping of the orientational distribution of the molccules in the rigid-limit spectra in this system, in contrast to the information obtained with the ${ }^{14} \mathrm{~N}-\mathrm{CSL}$ spectra.
\end{abstract}

\section{Introduction}

The dynamic structure of the lamellar phase of model membranes is often studied by probe techniques in which extraneous reporter molecules are embedded in the system. The observed spectroscopic properties of the probes then yield information about their orientational distribution and reorientational motion in the system. Electron spin paramagnetic resonance (EPR) has been widely used in such studies [1]. The spin-labelled derivative of $3 \alpha$-cholestane, 3-doxyl-cholestane (CSL),

1 Present address to which correspondence should be sent. has been found to be particularly useful in that its EPR spectra reflect the overall motion of the rigid molecules. The CSL molecules are incorporated into lipid bilayers with the steroid nucleus buried in the hydrophobic region and with the nitroxide group located near the polar heads of the lipids. As the major axis of the hyperfine tensor of the molecule is perpendicular to its long axis, the EPR spectrum of this spin probe is optimally sensitive to reorientational motions about its long axis.

The interpretation of the observed EPR spectra can be considerably simplified if macroscopically oriented samples (multibilayers) are used experimentally. Here the lamellar planes possess a com- 
mon, parallel, macroscopic orientation conveniently described by the common normal to their planes, the director. The use of such samples in EPR experiments affords the rotation of the director relative to the applied static magnetic field, so that a detailed study of the anisotropic properties of the spectra can be undertaken [2-8]. The model membrane system of dimyristoylphosphatidylcholine (DMPC) / cholesterol (molar ratio $2: 1$ ) is particularly convenient for such studies as it has been shown $[6,9]$ that the orientational order of the CSL molecules in this system is virtually indistinguishable from perfect alignment with their long axes along the bilayer normal, at temperatures in the region of the gel-lamellar phase transition of pure DMPC bilayers $\left(23^{\circ} \mathrm{C}\right)$. This behaviour has also been observed in other lamellar systems consisting of mixtures of saturated phosphatidylcholine molecules and cholesterol $[4,6]$. As a consequence of the perfect orientational order, the CSL molecules are restricted to undergo an axial rotational motion.

The EPR spectra of CSL molecules in these systems have usually been interpreted either on assuming fast motional narrowing $[2,3,6]$ or the rigid limit in which all motion has been quenched [4]. Saturation transfer EPR (ST EPR) studies of multibilayers of such systems, however, provided qualitative evidence for slow rotational motion of the CSL molecules [10,11]. A quantitative slow motion analysis of CSL in oriented low water content multibilayers of (pure) dipalmitoylphosphatidylcholine (DPPC) was given by Meirovitch and Freed [8]. However, this system exhibits a much lower degree of order than observed in the DMPC/cholesterol multibilayers.

Here we present a slow-motion analysis of the $\mathrm{X}$-band EPR spectra of CSL molecules embedded in multibilayers of the system DMPC/cholesterol $(2: 1)$. The experiments were performed with the isotopically substituted molecules ${ }^{15} \mathrm{~N}-\mathrm{CSL}\left(I=\frac{1}{2}\right)$ as well as the natural abundance probe ${ }^{14} \mathrm{~N}-\mathrm{CSL}$ $(I=1)$. The long axes of the CSL molecules are assumed to be perfectly ordered along the macroscopic director and to undergo either Brownian rotational diffusion or random jumps about their long molecular axes (planar rotor model). In the temperature range studied $\left(-18\right.$ to $\left.18^{\circ} \mathrm{C}\right)$ the random jump model provides a better description of the experimental spectra than the Brownian diffusion model. Using this random jump model, it has been possible to estimate motional rates for the cholestane probe in the lipid bilayer over a wide range of temperatures.

\section{Experimental}

\subsection{Sample preparation}

Dimyristoylphosphatidylcholine (DMPC) was obtained from Calbiochem, cholesterol from Sigma and ${ }^{14} \mathrm{~N}-\mathrm{CSL}$ (Label \#611) from Syva. ${ }^{15} \mathrm{~N}-\mathrm{CSL}$ was prepared by the procedure of Keana et al. [12], using the ${ }^{15} \mathrm{~N}$-2-amino-2-methyl-1-propanol prepared as described in ref. [13]. The molecular structures of the compounds are shown in fig. 1. Weighed amounts of DMPC and cholesterol were mixed with the appropriate amount of CSL in chloroform to form $(2: 1: 0.03)$ molar ratio mixtures for ${ }^{14} \mathrm{~N}$-CSL and $(2: 1: 0.015)$ for ${ }^{15} \mathrm{~N}$-CSL. The solvent was removed overnight under vacuum. The mixture was hydrated by equilibration with water vapour for $24 \mathrm{~h}$ above a saturated solution of $\mathrm{K}_{2} \mathrm{SO}_{4}$ (98\% relative humidity) at approximately $50^{\circ} \mathrm{C}$. The lipid material was oriented between glass plates $\left(0.2 \times 3 \times 8 \mathrm{~mm}^{3}\right)$ by the

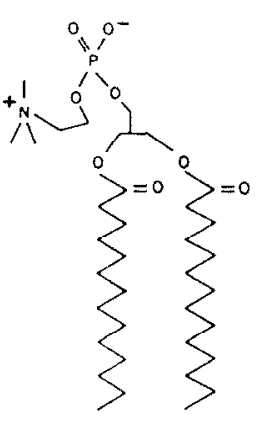

DMPC

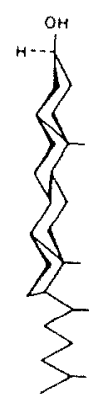

Cholesterol

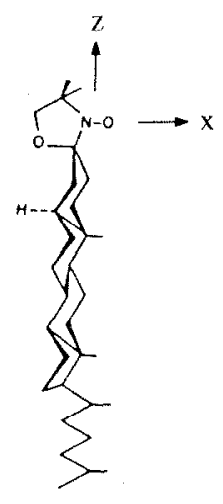

CSL
Fig. 1. Chemical compounds used in this work. The oxazolidine ring of CSL contains ${ }^{14} \mathrm{~N}(I=1)$ or ${ }^{15} \mathrm{~N}\left(I=\frac{1}{2}\right)$. The (average) orientation of the long axes of the molecules is parallel to the director $\boldsymbol{n}$ of the multibilayer. 
application of shear pressure. The macroscopic alignment was checked with a polarizing microscope equipped with a first-order red plate. The size of a typical sample built up of a stack of such sandwiches was about $4 \times 3 \times 8 \mathrm{~mm}^{3}$. After the samples had been oriented they were re-equilibrated with the vapour and subsequently again subjected to the alignment procedure. The sample was placed vertically on a support in a glass tube containing a saturated $\mathrm{K}_{2} \mathrm{SO}_{4}$ solution to maintain the hydration level during the experiments. The solution at the bottom of the tube, was placed well away from the active region of the EPR microwave cavity.

\subsection{EPR experiments}

Experiments utilizing the ${ }^{14} \mathrm{~N}$-CSL probes were carried out with a Varian E-9 X-band (3.2 kG)
EPR spectrometer equipped with a TM 110 cavity. Temperature control was achieved by passing nitrogen gas, precooled (liquid $\mathrm{N}_{2}$ ) and heated under control of a Varian V-4540 variable temperature accessory, over the sample. The temperature was measured with a copper-constantan thermocouple placed just above the sample, but outside the active region of the cavity. The accuracy of the temperature was $\pm 2^{\circ} \mathrm{C}$. Measurements on samples containing ${ }^{15} \mathrm{~N}-\mathrm{CSL}$ were performed with a Varian E-109 century series spectrometer. The temperature was regulated by an E-257 unit which brought precooled air (acetone/dry ice) to the desired temperature. The orientation of the sample director relative to the applied static magnetic field $\boldsymbol{H}_{0}$ was varied using a goniometer with an accuracy of $\pm 1^{\circ}$. The magnetic field modulation amplitude and microwave power were chosen sufficiently low as to avoid spectral distortion.

\section{Theory}

\subsection{The planar rotor model}

The EPR spectrum of a nitroxide radical placed in a magnetic field $\boldsymbol{H}_{0}$ is completely determined by the orientation of the magnetic tensors $g$ and $A$ relative to $\boldsymbol{H}_{0}$. As $\boldsymbol{H}_{0}$ is a symmetry axis of the magnetic interactions, it is conveniently chosen as the $z$ axis of the laboratory reference frame. The director $n$ of the uniaxial multibilayer sample is the symmetry axis for the orientational distribution and molecular rotational motion. Consequently the molecular orientation in the laboratory frame is described in terms of the composite transformation

$$
H_{0} \text { frame } \rightarrow n \text { frame } \rightarrow \text { molecular frame. }
$$

The molecules are assumed to undergo an axially symmetric reorientational motion about their long axes, taken to be the molecular $z$ axes. The principal axis systems of $\mathbf{g}$ and $\mathbf{A}$ are assumed to coincide and furthermore have one axis lying along the molecular $z$ axis. For the system under investigation we shall assume that the $z$ axes of the CSL molecules coincide with the director $n$. Therefore it is characterized by a single rotational degree of freedom. Let $\Omega \equiv\{\alpha, \beta, \phi\}$ be the set of Euler angles [14] defining the molecular orientation in the laboratory $\left(H_{0}\right)$ frame. The angles $\alpha$ and $\beta$ have a fixed value. We may set $\alpha=0$ since different values of $\alpha$ are magnetically equivalent and merely represent rotations around $\boldsymbol{H}_{0}$. The angle $\beta$ describes the orientation of the molecular $z$ axis (and hence $n$ ) relative to $H_{0}$. Its value $\beta=\theta$ is experimentally adjustable. The stochastic rotational motion on the other hand is characterized by $\phi$. This is depicted in fig. 2.

\subsection{Stochastic Liouville formalism}

Comprehensive discussions of the use of the stochastic Liouville formalism for the calculation of slow motion EPR lineshapes of spin labels in liquid crystals have been given by Freed and co-workers [15]. For 


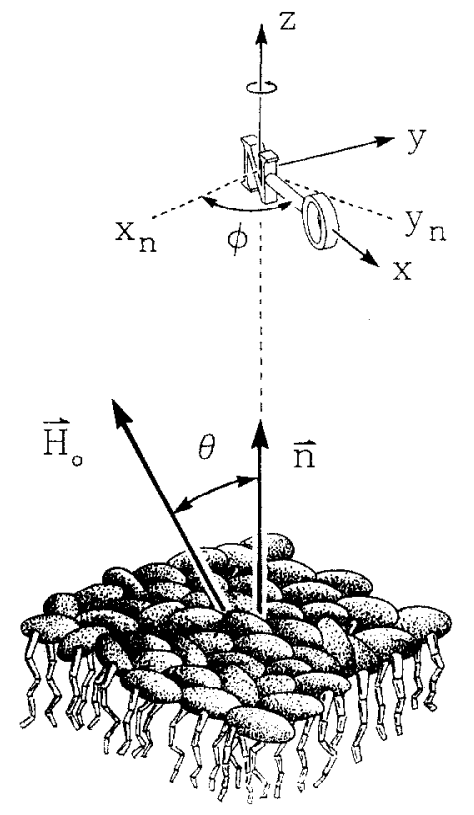

Fig. 2. Schematic illustration of the planar rotor model. The $x y z$ frame is fixed to the CSL molecule (cf. fig. 1). The director $\boldsymbol{n}$ of the multibilayer defines the $z$ axis of the director frame, which is supplemented by $x_{n}$ and $y_{n}$. The molecular $z$ axis is aligned with $n$. Rotation of the molecule about the director is defined by the angle $\phi$. The angle $\theta$ between $n$ and the magnetic field $\boldsymbol{H}_{0}$ has a fixed value.

our case where the CSL molecules are aligned along the director $n$, which in turn has a fixed orientation relative to $\boldsymbol{H}_{0}$, the description can be considerably simplified. The transition from the general three-dimensional formulation of the stochastic Liouville equation (SLE) to the present one-dimensional model can be accomplished by writing the equilibrium orientational distribution $P_{0}(\Omega)$ as

$$
P_{0}(\Omega)=\delta(\alpha)(\sin \beta)^{-1} \delta(\beta-\theta) p_{0}(\phi),
$$

where a reduced distribution function $p_{0}(\phi)$ has been introduced. Consider some function $F(\Omega)$ whose ensemble average

$$
\langle F\rangle=\int \mathrm{d} \Omega F(\Omega) P_{0}(\Omega)
$$

is of interest. On using eq. (1) we have

$$
\langle F\rangle=\int \mathrm{d} \alpha \int \mathrm{d} \beta \sin \beta \int \mathrm{d} \phi F(\alpha, \beta, \phi) P_{0}(\alpha, \beta, \phi)=\int \mathrm{d} \phi F(0, \theta, \phi) p_{0}(\phi) .
$$

This effectively implies the substitutions $F(\Omega) \rightarrow F(0, \theta, \phi)$ and $P_{0}(\Omega) \rightarrow p_{0}(\phi)$. With this prescription we obtain for $\mathscr{P}(\omega)$, the power absorbed ${ }^{\# 1}$ from a microwave field with frequency $\omega / 2 \pi[16]$,

$$
\mathscr{P}(\omega) \propto \operatorname{Im}\left\langle p_{0}^{1 / 2}(\phi) S_{-}\left|\left[\omega+\mathscr{H}(\phi)^{\times}-\mathrm{i}\left(\Gamma_{\phi}+\Gamma_{R}\right)\right]^{-1}\right| p_{0}^{1 / 2}(\phi) S_{-}\right\rangle
$$

where a scalar product is defined as

$$
\langle\mathscr{A}(\phi) \mid \mathscr{B}(\phi)\rangle=\int \mathrm{d} \phi \operatorname{Tr}\left[\mathscr{A}^{\dagger}(\phi) \mathscr{B}(\phi)\right] .
$$

\#1 Experimentally the first derivative of $\mathscr{P}(\omega)$ with respect to the static magnetic field is detected. 
Here $\mathscr{H}(\phi)$ is the spin Hamiltonian (the commutator superoperator notation $A^{\times} B \equiv[A, B]$ is employed). $\Gamma_{\phi}$ is the stochastic rotation operator corresponding to the planar rotor model. The expression given for $\mathscr{P}(\omega)$ requires $\Gamma_{\phi}$ to be replaced by its symmetric form $p_{0}^{-1 / 2}(\phi) \Gamma_{\phi} p_{0}^{1 / 2}(\phi)$ [17]. However, as $p_{0}(\phi)$ is isotropic (see below), this symmetrization does not affect the calculations. $\Gamma_{\mathrm{R}}$ is a quantum mechanical operator accounting for the residual spin relaxation processes, i.e. those not attributed to the stochastic modulation of $\mathscr{H}(\phi)$. Furthermore $S_{-}$is the electron spin lowering operator.

In order to apply any of the iterative numerical techniques currently available for the calculation of (4) [18-21], we need to construct a basis for the representation of the operators. As the molecular properties are assumed to be invariant under a $180^{\circ}$ rotation of the molecular $z$ axis, a convenient symmetry-adapted basis set in $\phi$ space is formed by

$$
g_{n}(\phi)=(1 / 2 \pi)^{1 / 2} \mathrm{e}^{-\mathrm{in} \phi}, \quad n \text { even. }
$$

Hence the full basis set, including spin, for this case (where the high-field approximation and the no-saturation condition are implied) is

$$
\left|n ; \nu \nu^{\prime}\right\rangle=g_{n}(\phi)|\beta \nu\rangle\left\langle\alpha \nu^{\prime}\right|,
$$

where $\nu$ and $\nu^{\prime}$ are the nuclear spin indices and $\beta(\alpha)$ denotes the electron spin quantum number $m_{S}=-\frac{1}{2}$ $\left(\frac{1}{2}\right)$. From our assumption of molecular axial symmetry we have

$$
p_{0}(\phi)=(1 / 2 \pi)
$$

and consequently

$$
\left\langle n ; \nu \nu^{\prime} \mid p_{0}^{1 / 2}(\phi) S_{-}\right\rangle=\delta_{\nu, v^{\prime}} \int \mathrm{d} \phi g_{n}(\phi)^{*} p_{0}(\phi)=\delta_{p,}, \delta_{n 0}
$$

The rotation operator for the Brownian diffusion model is given by [22,23]

$$
\Gamma_{\phi}=-D_{\|} \partial^{2} / \partial \phi^{2}
$$

where $D_{\|}$is the rotational diffusion coefficient for rotation about the molecular $z$ axis. Its matrix elements are given by

$$
\left\langle n_{1} ; \nu_{1} \nu_{1}^{\prime}\left|\Gamma_{\phi}\right| n_{2} ; \nu_{2} \nu_{2}^{\prime}\right\rangle=\delta_{\nu_{1} \nu_{2} \nu_{1}^{\prime} \nu_{2}^{\prime}} \delta_{n_{1} n_{2}} D_{\|} n_{1}^{2} .
$$

The integral operator describing the random jump model is given by [23]

$$
\Gamma_{\phi}=\frac{1}{\tau} \int \mathrm{d} \phi^{\prime}\left[\delta\left(\phi-\phi^{\prime}\right)-p_{0}(\phi)\right]=\frac{1}{\tau} \int \mathrm{d} \phi^{\prime}\left[\delta\left(\phi-\phi^{\prime}\right)-1 / 2 \pi\right],
$$

where $\tau$ is the mean residence time in a particular orientation. Its matrix elements are given by

$$
\left\langle n_{1} ; \nu_{1} \nu_{1}^{\prime}\left|\Gamma_{\phi}\right| n_{2} ; \nu_{2} \nu_{2}^{\prime}\right\rangle=\delta_{\nu_{1} \nu_{2} \nu_{1} \nu_{2}^{\prime}} \delta_{n_{1} n_{2}} \tau^{-1}\left\{1-\delta_{n_{1} 0}\right\}
$$

The spin Hamiltonian $\mathscr{H}(\phi)$ for a nitroxide radical is obtained from the more general form $\mathscr{H}(\Omega)$ [16] on replacing $\Omega$ by $\{0, \theta, \phi\}$. This yields in the high field approximation and under the assumption of coinciding principal axes of $\mathbf{g}$ and $\mathbf{A}$ :

$$
\begin{aligned}
\mathscr{H}(\phi)= & \left(\beta_{\mathrm{e}} / \hbar\right) g^{(\mathrm{iso})} H_{0} S_{z}-\gamma_{\mathrm{e}} a^{(\mathrm{iso})} I_{z} S_{z}-\gamma_{\mathrm{n}} H_{0} I_{z}+\sum_{p} d_{0 p}^{2}(\theta)\left[F_{p}^{\prime}+A_{p}^{\prime} I_{z}\right] \mathrm{e}^{-\mathrm{i} p \phi} S_{z} \\
& +\sum_{p} A_{p}\left[d_{1 p}^{2}(\theta) I_{+}-d_{-1 p}^{2}(\theta) I_{-}\right] \mathrm{e}^{-\mathrm{i} p \phi} S_{z},
\end{aligned}
$$


where the summations are restricted to $p=0, \pm 2$. The following reduced Wigner rotation matrix elements [14] are employed:

$$
d_{m n}^{\mathrm{L}}(\theta) \equiv \mathscr{D}_{m n}^{\mathrm{L}}(0, \theta, 0)
$$

$\gamma_{\mathrm{c}}$ and $\gamma_{\mathrm{n}}$ are the electronic and nuclear (nitrogen) gyromagnetic ratios. Furthermore $g^{(\text {iso })}=\frac{1}{3} \operatorname{Tr}(\mathbf{g})$ and $A^{(i s o)}=\frac{1}{3} \operatorname{Tr}(\mathbf{A})$. The quantities $F_{p}^{\prime}$ and $\left(A_{p}, A_{p}^{\prime}\right)$ are (within a constant factor) spherical tensor components of $\mathbf{g}$ and $\mathbf{A}$ respectively (see appendix). The matrix elements of $\mathscr{H}(\phi)^{\times}$are

$$
\begin{aligned}
\left\langle n_{1} ;\right. & \left.\nu_{1} \nu_{1}^{\prime}\left|\mathscr{H}(\phi)^{\times}\right| n_{2} ; \nu_{2} \nu_{2}^{\prime}\right\rangle \\
= & -\delta_{n_{1} n_{2}} \delta_{\nu_{1} \nu_{1}^{\prime}} \delta_{\nu_{2} \nu_{2}^{\prime}}\left\{\left|\gamma_{e}\right|\left[H_{0}+\frac{1}{2} a^{(i s o)}\left(\nu_{1}+\nu_{1}^{\prime}\right)\right]+\gamma_{n} H_{0}\left(\nu_{1}-\nu_{1}^{\prime}\right)\right\} \\
& -\delta_{\nu_{1} \nu_{2}} \delta_{\boldsymbol{\nu}_{1}^{\prime} \nu_{2}^{\prime}} \sum d_{p} d_{0 p}^{2}(\theta)\left[F_{p}^{\prime}+\frac{1}{2} A_{p}^{\prime}\left(\nu_{1}+\nu_{1}^{\prime}\right)\right] \delta_{n_{1}, p+n_{2}}-\frac{1}{2} \sum_{p} A_{p} \delta_{n_{1}, p+n_{2}} \sum_{q} d_{q p}^{2}(\theta) q \\
& \times\left[K\left(\nu_{1}, \nu_{2}\right) \delta_{\nu_{1}, \nu_{2}+q^{\prime}} \delta_{\nu_{1}^{\prime}, \nu_{2}^{\prime}}+K\left(\nu_{1}^{\prime}, \nu_{2}^{\prime}\right) \delta_{\nu_{1} \nu_{2}} \delta_{\nu_{1}^{\prime}, \nu_{2}^{\prime}-q}\right],
\end{aligned}
$$

where $K\left(\nu_{1}, \nu_{2}\right)=\left[I(I+1)-\nu_{1} \nu_{2}\right]^{1 / 2}, \dot{p}=0, \pm 2$ and $q= \pm 1$

Finally, the relaxation operator $\Gamma_{\mathrm{R}}$ has matrix elements

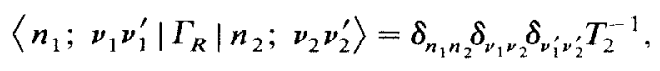

where $T_{2}$ is an electron spin-spin relaxation time.

Summarizing, we have given the representations of $\left|p_{0}^{1 / 2}(\phi) S_{-}\right\rangle, \mathscr{H}(\phi)^{\times}, \Gamma_{\phi}$ and $\Gamma_{\mathrm{R}}$. This provides the basis for the calculation of $\mathscr{P}(\omega)$ (eq. (4)). The spectral simulations which will be discussed below were carried out using Padé approximants [19], although the Lanczos algorithm [18] can also be used. The numerical problems associated with the applications of Padé approximant techniques [21] were not encountered in these simulations.

\section{Results and discussion}

\subsection{General considerations}

Typical spectra of ${ }^{14} \mathrm{~N}-\mathrm{CSL}$ embedded in DMPC/cholesterol multibilayers oriented with the director parallel $\left(\theta=0^{\circ}\right)$ and perpendicular $(\theta=$ $\left.90^{\circ}\right)$ to the magnetic field direction are shown in fig. 3 . These spectra provide experimental justification for the planar rotor model. Consider a single nitroxide radical with a fixed orientation $(\theta, \phi)$ relative to the magnetic field $H_{0}$ (fig. 2). Its EPR spectrum can be described to a good approximation by a superposition of $(2 I+1)$ lines, each one characterized by a value of the nuclear magnetic quantum number $v=-I,-I+1, \ldots, I$. These are centred at the frequencies [24]

$$
\begin{aligned}
& \omega_{\text {res }}(\theta, \phi, v) \\
& \quad=\left|\gamma_{\mathrm{e}}\right|\left\{\left[g(\theta, \phi) / g^{\text {(iso) }}\right] H_{0}+v A(\theta, \phi)\right\},
\end{aligned}
$$

where

$$
g(\theta, \phi)=\left(g_{x} \cos ^{2} \phi+g_{y} \sin ^{2} \phi\right) \sin ^{2} \theta+g_{z} \cos ^{2} \theta
$$

and

$$
\begin{aligned}
& A(\theta, \phi) \\
& \quad=\left[\left(A_{x}^{2} \cos ^{2} \phi+A_{y}^{2} \sin ^{2} \phi\right) \sin ^{2} \theta+A_{z}^{2} \cos ^{2} \theta\right]^{1 / 2} .
\end{aligned}
$$

Typical values of the tensor elements for ${ }^{14} \mathrm{~N}-\mathrm{CSL}$ are

$\mathbf{g}=\operatorname{diag}\left(g_{x}, g_{y}, g_{z}\right) \approx \operatorname{diag}(2.008,2.002,2.006)$

and

$$
\mathbf{A}=\operatorname{diag}\left(A_{x}, A_{y}, A_{z}\right) \approx \operatorname{diag}(6,33,6) \mathrm{G} .
$$

Consider now an ensemble of perfectly aligned CSL molecules as implied in the planar rotor model. For the case of $\theta=0^{\circ}\left(\boldsymbol{H}_{0} \| \boldsymbol{n}\right)$ the EPR 


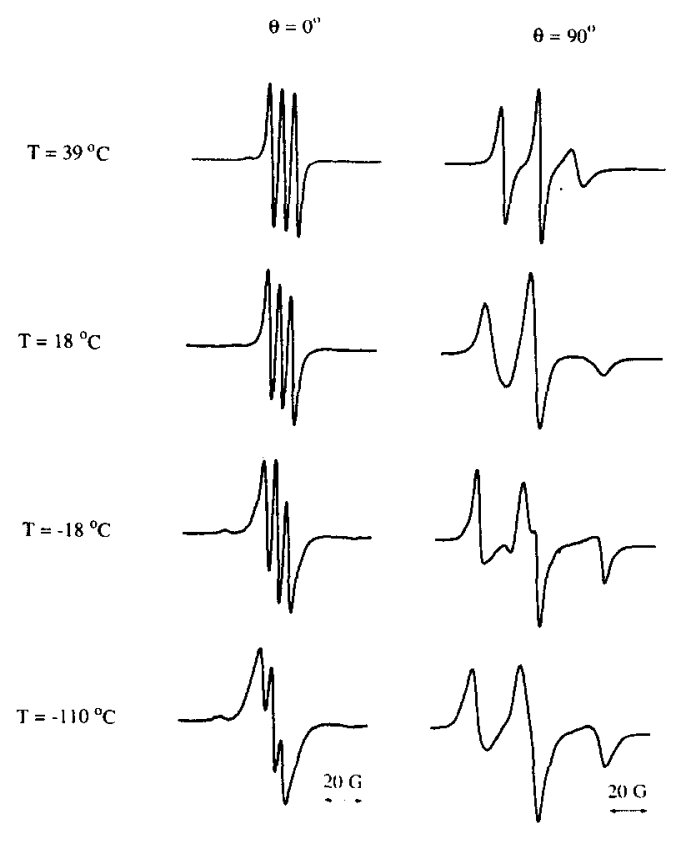

Fig. 3. EPR spectra of ${ }^{14} \mathrm{~N}-\mathrm{CSL}$ in DMPC/cholesterol $(2: 1)$ bilayers, recorded at various temperatures with $\theta=0^{\circ}\left(H_{0} \| n\right)$ and $\theta=90^{\circ}\left(\boldsymbol{H}_{0} \perp n\right)$ respectively. Each temperature represents a motional regime: $T=39^{\circ} \mathrm{C}$ : motional narrowing; $T=18^{\circ} \mathrm{C}$ : slow motion; $T=-18^{\circ} \mathrm{C}$ : rigid limit; $T=$ $-110^{\circ} \mathrm{C}$ : rigid limit with dominant broadening.

spectrum consists of three lines with a spacing $A_{z}$ and with its centre at $\omega_{\text {res }}=\left|\gamma_{\mathrm{e}}\right|\left(g_{z} / g^{(\text {iso })}\right) H_{0}$. The EPR spectrum for $\theta=90^{\circ}\left(\boldsymbol{H}_{0} \perp \boldsymbol{n}\right)$ on the other hand will be more complex as it reflects the distribution of orientations, $\phi$, about the director $n$ (see eqs. (17) and (18a), (18b)). Rotational motion about the $z$-axis has no effect on the spectral lineshape for $\theta=0^{\circ}$, however the complex $\theta=90^{\circ}$ spectrum will change markedly in the presence of motion, reducing to a triplet with a spacing $\frac{1}{2}\left(A_{x}\right.$ $\left.+A_{y}\right)$ if the motion is sufficiently fast. Deviations from this ideal behaviour as a result of a static orientational distribution of the molecular $z$ axes about the director $n$ will particularly affect the spectral lineshape for $\theta=0^{\circ}$. This arises from the contribution of a large component of the hyperfine tensor $A_{y} \approx 33 \mathrm{G}$, to a spectrum essentially determined by a small component, $A_{z} \approx 6 \mathrm{G}$.

The experimental line spacing $A_{\|}$for $\theta=0^{\circ}$ is found to increase only slightly over the temperature range studied (fig. 4). In contrast marked

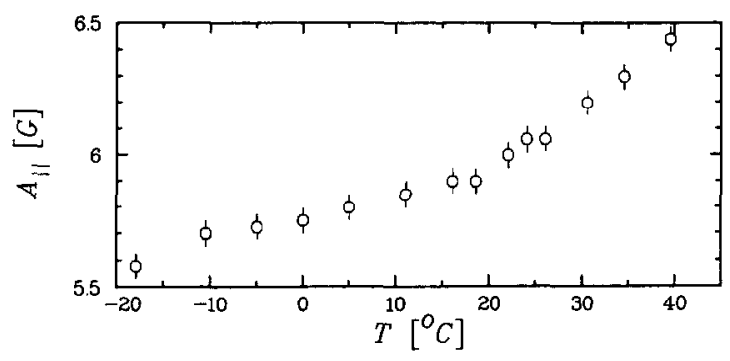

Fig. 4. Experimental line splittings $A_{\|}$of the EPR spectra of ${ }^{14} \mathrm{~N}-\mathrm{CSL}$, recorded with $\theta=0^{\circ}\left(\boldsymbol{H}_{0} \| n\right)$, as a function of temperature.

changes are observed in the spectra for $\theta=90^{\circ}$ over the same temperature range (fig. 3). These changes indicate that the motional rates increase for values characteristic of the rigid limit to the fast motional region. Consequently we have good grounds for using the planar rotor model. Nevertheless we note that at low temperatures the $\theta=0^{\circ}$ spectra are considerably broadened, but with no significant shift in the positions of the outer peaks. This effect is similar to the one observed by Marsh and Smith [25] in multibilayers of a similar system, containing high CSL concentrations, at room temperature. Thus we attribute these observations to the effects of electron spin-spin (exchange) interactions and not to the decrease in order as suggested in ref. [4]. At low temperatures the lateral translational mobility of CSL over the lamellar planes is highly restricted and our observations point to the formation of clusters of CSL molecules. The appearance of "shoulders" at intermediate temperatures suggests the co-existence of clusters of interacting CSL molecules with a population of "free" molecules. A further increase in temperature shifts the equilibrium towards the "free" state. Finally we note that the broadening effects described here cannot be explained by invoking two conformations of the oxazolidine ring [26].

\subsection{Rigid limit}

The low-temperature broadening of the spectra of the CSL molecules hampers the accurate determination of the components of the magnetic interaction tensors from the rigid-limit spectrum. 
Thus one is forced to seek a spectrum for which the motion is slow enough and the broadening as small as possible to serve as an effective rigid-limit case. The $\theta=90^{\circ}$ spectrum for $T=-18^{\circ} \mathrm{C}$ was found to be a suitable compromise. This is in accord with the findings of Hemminga for DPPc/cholesterol multibilayers [4]. The $z$ component of A was determined directly from the line splitting of the broadened $\theta=0^{\circ}$ spectra at the same temperature. In order to obtain the $x$ and $y$ components of $\mathbf{g}$ and $\mathbf{A}$, however, complete spectral simulations were required. We found it convenient to compute the lineform of the $\theta=90^{\circ}$ spectra in the rigid limit directly as a superposition of spectral lines whose positions and spacings are given by eqs. (17) and (18a), (18b), instead of solving the SLE. In this way angle-dependent intrinsic lineshapes, other than Lorentzian, can be included in a straightforward way in contrast to the SLE formalism. For the calculation of the $\theta=90^{\circ}$ rigid-limit spectra eqs. (17) and (18a), $(18 \mathrm{~b})$ are reduced to

$$
\begin{aligned}
H_{\mathrm{res}}(\phi, v) & \equiv \omega_{\mathrm{res}}\left(90^{\circ}, \phi, v\right) /\left|\gamma_{\mathrm{e}}\right| \\
& =\left[g_{\perp}(\phi) / g^{\text {(iso) }}\right] H_{0}+v A_{\perp}(\phi),
\end{aligned}
$$

where

$g_{\perp}(\phi)=g_{x} \cos ^{2} \phi+g_{y} \sin ^{2} \phi$

and

$A_{\perp}(\phi)=\left(A_{x}^{2} \cos ^{2} \phi+A_{y}^{2} \sin ^{2} \phi\right)^{1 / 2}$.

The contribution to the spectrum from this resonance position is obtained by attributing some intrinsic lineshape to the resonance positioned at $H_{\text {res }}(\phi, v)$. Subsequently a summation over $v$ and an integration (numerically a summation) over $\phi$ is performed, assuming $p_{0}(\phi)$ to be a uniform distribution (see eq. (7)). The intrinsic lineshape was chosen to be a convolution of a Lorentzian line and a $\phi$-dependent Gaussian line. The absorption lineshape is given by

$$
\begin{gathered}
\mathscr{L}(H)=\sum_{\nu} \int \mathrm{d} \phi \int \mathrm{d} H^{\prime} f_{\mathrm{G}}\left(H-H^{\prime}, \phi\right) \\
\times f_{\mathrm{L}}\left(H^{\prime}-H_{\text {res }}(\phi, v)\right)
\end{gathered}
$$

and its experimentally detected first derivative

$$
\begin{aligned}
\frac{\mathrm{d} \mathscr{L}(H)}{\mathrm{d} H}= & \sum_{v} \int \mathrm{d} \phi \int \mathrm{d} H^{\prime} \frac{\mathrm{d}}{\mathrm{d} H} f_{\mathrm{G}}\left(H-H^{\prime}, \phi\right) \\
& \times f_{\mathrm{L}}\left(H^{\prime}-H_{\mathrm{res}}(\phi, v)\right)
\end{aligned}
$$

where a Lorentzian lineshape

$f_{\mathrm{L}}(\Delta H)=\frac{1}{\pi}\left|\gamma_{\mathrm{e}}\right| T_{2}\left(\frac{1}{1+\gamma_{\mathrm{e}}^{2} T_{2}^{2}(\Delta H)^{2}}\right)$

and a Gaussian lineshape

$f_{\mathrm{G}}(\Delta H, \phi)=\frac{1}{\sigma(\phi) \pi^{1 / 2}} \exp \left(\frac{-(\Delta H)^{2}}{2 \sigma(\phi)^{2}}\right)$

with

$\sigma(\phi)=\left(\sigma_{x}^{2} \cos ^{2} \phi+\sigma_{y}^{2} \sin ^{2} \phi\right)^{1 / 2}$

were introduced.

Good fits for the $\theta=90^{\circ}$ spectra of ${ }^{14} \mathrm{~N}-\mathrm{CSL}$ and ${ }^{15} \mathrm{~N}$-CSL respectively were obtained with the parameters given in table 1 , as is illustrated in fig. 5. As we did not carry out cxact field/frequency calibrations the absolute values of the $g$ components may not be exact. However, for the $\theta=90^{\circ}$ spectra only the difference $g_{x}-g_{y}$ is relevant. In addition we note that we found $\mid A_{x}\left({ }^{15} \mathrm{~N}\right) /$ $A_{x}\left({ }^{14} \mathrm{~N}\right) \mid=1.56$, in contrast to $\mid A_{y}\left({ }^{15} \mathrm{~N}\right) /$ $A_{y}\left({ }^{14} \mathrm{~N}\right) \mid=1.40$. This ratio is expected to be 1.40 merely on account of the gyromagnetic ratios of ${ }^{14} \mathrm{~N}$ and ${ }^{15} \mathrm{~N}$. However, changes in bond angles

Table 1

Magnetic and broadening parameters obtained from the rigidlimit simulations of ${ }^{14} \mathrm{~N}$-CSL and ${ }^{15} \mathrm{~N}$-CSL

\begin{tabular}{lccl}
\hline \multicolumn{5}{c}{${ }^{14} \mathrm{~N}-\mathrm{CSL}$} & ${ }^{15} \mathrm{~N}-\mathrm{CSL}$ \\
\hline$g_{x}$ & 2.0081 & 2.0081 & \\
$g_{y}$ & 2.0024 & 2.0024 & \\
$g_{z}$ & 2.0061 & 2.0061 & \\
$A_{x}(\mathrm{G})$ & 6.4 & -10 & $(1.56)^{\mathrm{a})}$ \\
$A_{y}(\mathrm{G})$ & 33.6 & -47 & $(1.40)^{\mathrm{a})}$ \\
$A_{z}(\mathrm{G})$ & 5.6 & -8.3 & $(1.48)^{\mathrm{a})}$ \\
$\sigma_{x}(\mathrm{G})$ & 1.5 & 1.6 & \\
$\sigma_{y}(\mathrm{G})$ & 1 & 1.2 & \\
$T_{z}(\mathrm{~s})$ & $3 \times 10^{-8}$ & $3 \times 10^{-8}$ & \\
\hline
\end{tabular}

a) Value in parentheses denotes $\left|A_{i}\left({ }^{15} \mathrm{~N}\right) / A_{i}\left({ }^{14} \mathrm{~N}\right)\right|$, wherc $i=x, y$ or $z$. 

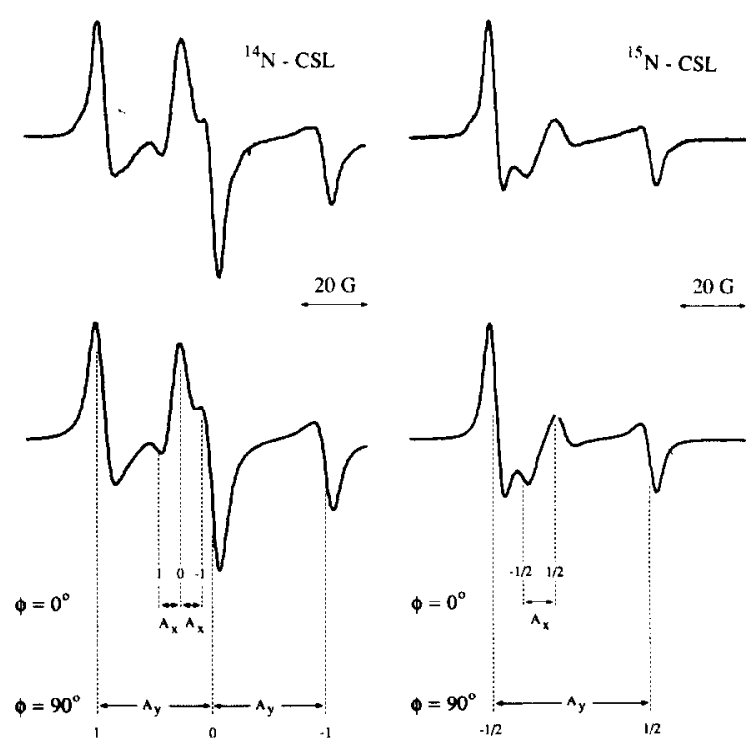

Fig. 5. Experimental (top) and calculated (bottom) rigid limit spectra of ${ }^{14} \mathrm{~N}$-CSL (left) and ${ }^{15} \mathrm{~N}$-CSL (right) for $\theta=90^{\circ}$. The experimental spectra were recorded at $T=-18^{\circ} \mathrm{C}$. The parameters used in the simulations are given in table 1 . Spectral positions of the $\left(\phi=0^{\circ}\right)$ multiplet (line spacing $\left.A_{x}\right)$ and ( $\phi=90^{\circ}$ ) multiplet (line spacing $A_{y}$ ), as defined by eq. (19), are indicated. Each line is characterized by its nuclear magnetic quantum number $v:(-1,0,1)$ for ${ }^{14} \mathrm{~N}-\mathrm{CSL}$ and $\left(-\frac{1}{2}, \frac{1}{2}\right)$ for ${ }^{15} \mathrm{~N}-\mathrm{CSL}$.

and bond lengths upon isotopic substitution, resulting in changes of $\mathbf{A}$, cannot be ruled out a priori. Therefore, we prefer to adhere to the $A$ components obtained from our rigid-limit simulations. Note that the $v=-\frac{1}{2}$ and $v=\frac{1}{2}$ regions of the ${ }^{15} \mathrm{~N}$-CSL spectrum do not overlap. Consequently each spectral position corresponds uniquely to a particular value of $\phi\left(\bmod \left(180^{\circ}\right)\right)$. Further aspects concerning the origin and reliability of the various parameters will now be discussed.

\subsubsection{Gaussian broadening}

Luckhurst et al. [27] reported a detailed analysis of electron-proton couplings in CSL, responsible for the inhomogeneous broadening of the (fast motional) EPR absorption lines. The systems studied were a smectic A liquid crystal and a thiourea crystal. As a result of the highly anisotropic environment only partial averaging of the interaction takes place, resulting in effectively axially symmetric hyperfine tensors for the various sets of equivalent protons, where the symmetry axis is the molecular $z$ axis. We used the transverse components of these tensors to calculate a single line taking a Lorentzian line with $T_{2}=3 \times$ $10^{-8} \mathrm{~s}$ (cf. eq. (23)) as the homogeneous lineshape. A perfect fit of the resultant lineshape was obtained by a convolution of the same Lorentzian line with a Gaussian line having $\sigma=1.1 \mathrm{G}$ (cf. eq. (24)). This result is strictly applicable to the $\theta=$ $90^{\circ}$ spectrum in the motional narrowing region only. However, the values of $\sigma_{x}$ and $\sigma_{y}$ found here for the rigid limit by trial and error (cf. table 1) are quite similar and therefore reasonable and acceptable. A similar Gaussian fit procedure was applied to the (effective) proton coupling tensor components parallel to the molecular $z$ axis. This yielded $\sigma_{z}=1.05 \mathrm{G}$. However, the additional broadening present at low temperatures distorts the $\theta=90^{\circ}$ spectra to such an extent that confirmation of this parameter was not possible. On the other hand we note that at high temperatures, where this broadening is virtually absent, a value $\sigma_{z}=0.8 \mathrm{G}$ appeared to give satisfactory results, so again a reasonably consistent picture was obtained.

\subsubsection{Lorentzian broadening}

The spin-spin relaxation time $T_{2}=3 \times 10^{-8} \mathrm{~s}$ accounts for relaxation processes which are not caused by rotational modulation of $\mathscr{H}(\phi)$. None of the other parameters employed in the simulations can be varied as to compensate for a different choice of $T_{2}$. Moreover, Meirovitch [26] found for CSL in thiourea a value $\left(\left|\gamma_{e}\right| T_{2}\right)^{-1}=1.5 \mathrm{G}$, which corresponds to $T_{2}=3.8 \times 10^{-8} \mathrm{~s}$, over a large temperature range $\left(-58\right.$ to $\left.80^{\circ} \mathrm{C}\right)$. We thus believe that the value $T_{2}=3 \times 10^{-8} \mathrm{~s}$ (cf. table 1) is acceptable and do not expect it to change appreciably at higher temperatures.

\subsection{Slow motion}

The $\theta=90^{\circ}$ spectra of CSL in the temperature range $-18 \leqslant T \leqslant 18^{\circ} \mathrm{C}$ were simulated by solving the stochastic Liouville equation as discussed in section 3.2. The lower bound was discussed in 


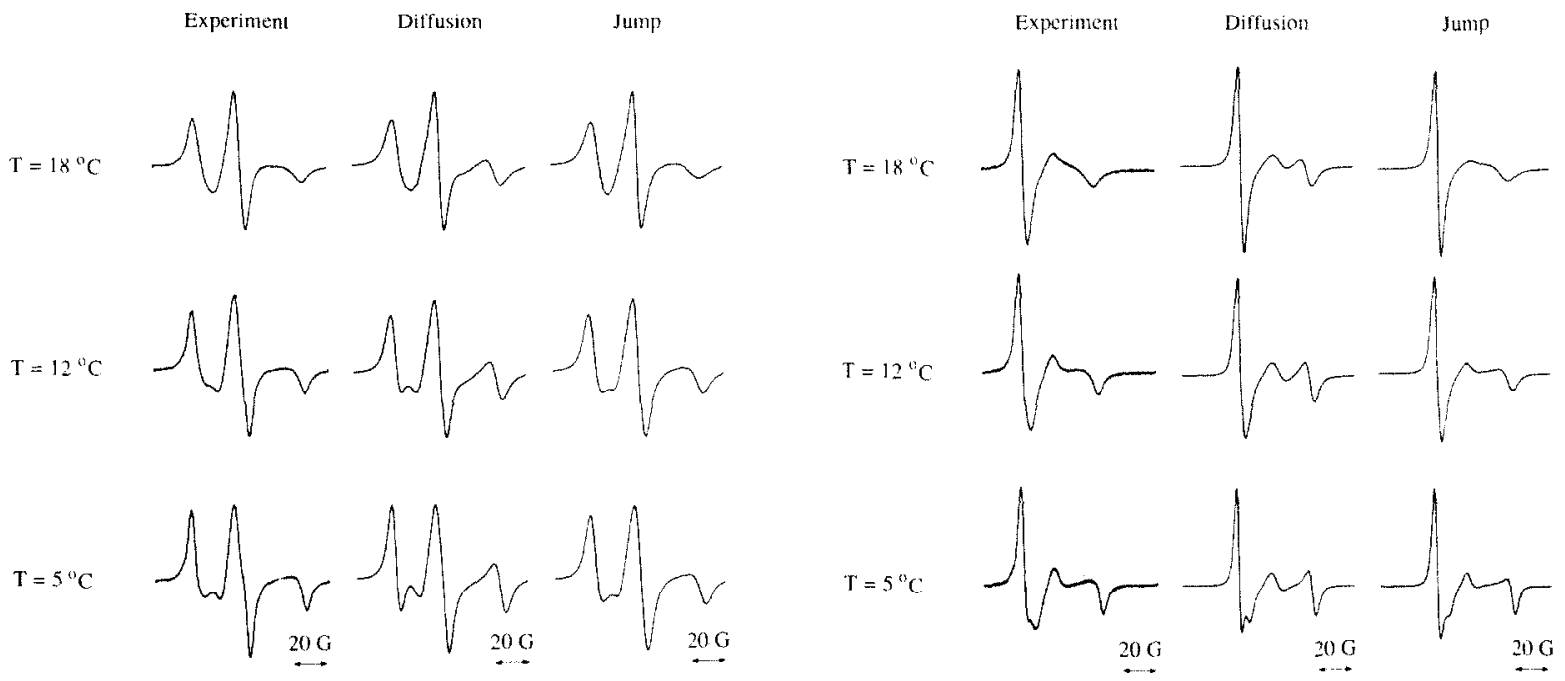

Fig. 6. Experimental and calculated EPR spectra of ${ }^{14}$ N-CSL for $\theta=90^{\circ}$. Experimental spectra were recorded at $T=5,12$ and $18^{\circ} \mathrm{C}$ respectively. The calculations were performed with the static parameters given in table 1 , except for an isotropic Gaussian broadening with $\sigma=0.8 \mathrm{G}$. The motional parameters employed in the brownian diffusion model and the random jump model are given in table 2.

relation to the interpretation of rigid-limit spectra. The upper bound was set by the behaviour of $A_{\|}$, i.e. the line splitting for $\theta=0^{\circ}$, as given in fig. 4 for ${ }^{14} \mathrm{~N}$-CSL. As can be seen the increase of $\mathrm{A}_{11}$ with temperature is more pronounced at $T \geqslant 20^{\circ} \mathrm{C}$ than at lower temperatures. This implies that the planar rotor model is losing its validity quite rapidly in this region, i.e. rotational motion of the molecular $z$ axis with respect to $n$ should be included in the description. Moreover for $T \geqslant$ $20^{\circ} \mathrm{C}$ the rotational motional rates correspond to the fast motion (Redfield) regime and conse-
Fig. 7. Experimental and calculated EPR spectra of ${ }^{15} \mathrm{~N}-\mathrm{CSL}$ for $\theta=90^{\circ}$. Experimental spectra were recorded at $\mathrm{T}=5^{\circ} \mathrm{C}$, $12^{\circ} \mathrm{C}$ and $18^{\circ} \mathrm{C}$ respectively. The calculations were performed with the static parameters given in Table 1 , except for an isotropic Gaussian broadening with $\sigma=0.8 \mathrm{G}$. The motional parameters employed in the Brownian diffusion model and the random jump model are given in Table 2.

quently all reorientational models will produce the same spectral lineshapes. The values of the tensor components and $T_{2}$ given in table 1 were used for the computations. The calculated spectra were convoluted with an isotropic Gaussian lineshape with $\sigma=0.8 \mathrm{G}$ to account for inhomogeneous broadening. The slow motion lineshapes, however, were not significantly changed by this broadening. Both the small-step Brownian diffusion and the random jump models were used in the simulations. It must be emphasized that each model is characterized by a single adjustable parameter, i.e.

Table 2

Motional parameters used in the spectral simulations of ${ }^{14} \mathrm{~N}-\mathrm{CSL}$ and ${ }^{15} \mathrm{~N}-\mathrm{CSL}$

\begin{tabular}{|c|c|c|c|c|c|c|}
\hline \multirow{2}{*}{$\begin{array}{l}T \\
\left({ }^{\circ} \mathrm{C}\right)\end{array}$} & \multicolumn{3}{|c|}{ Brownian diffusion } & \multicolumn{3}{|c|}{ Random jump } \\
\hline & $\begin{array}{l}D_{\|}\left({ }^{14} \mathrm{~N}\right) \\
\left(\mathrm{s}^{-1}\right)\end{array}$ & $\begin{array}{l}D_{\|}\left({ }^{15} \mathrm{~N}\right) \\
\left(\mathrm{s}^{-1}\right)\end{array}$ & $\begin{array}{l}D_{\|}\left({ }^{15} \mathrm{~N}\right) \\
D_{i \mid}\left({ }^{14} \mathrm{~N}\right)\end{array}$ & $\begin{array}{l}\tau\left({ }^{14} \mathrm{~N}\right) \\
(\mathrm{s})\end{array}$ & $\begin{array}{l}\tau\left({ }^{15} \mathrm{~N}\right) \\
(\mathrm{s}) \\
\end{array}$ & $\begin{array}{l}\tau\left({ }^{14} \mathrm{~N}\right) / \\
\tau\left({ }^{15} \mathrm{~N}\right)\end{array}$ \\
\hline 18 & $4 \times 10^{7}$ & $3.5 \times 10^{7}$ & 0.9 & $5.5 \times 10^{-9}$ & $6 \times 10^{-9}$ & 0.9 \\
\hline 12 & $2.5 \times 10^{7}$ & $2 \times 10^{7}$ & 0.8 & $1 \times 10^{-8}$ & $1.2 \times 10^{-8}$ & 0.8 \\
\hline 5 & $1.5 \times 10^{7}$ & $1 \times 10^{7}$ & 0.7 & $1.2 \times 10^{-8}$ & $2.4 \times 10^{-8}$ & 0.5 \\
\hline
\end{tabular}


the motional rate. Reproduction of the separation of the outer peaks of the spectrum, which was found to decrease monotonically with increasing motion, was used as a constraint for the acceptance of a simulation. However, only by considering the shape of the complete spectrum conclusions were drawn in favour of a particular rotational model.

Results for ${ }^{14} \mathrm{~N}$-CSL and ${ }^{15} \mathrm{~N}-\mathrm{CSL}$ at three temperatures are shown in figs. 6 and 7 respectively. The corresponding motional parameters, $D_{\|}$or $\tau$, are given in table 2. Spectral simulations using the random jump model were found to fit the experimental spectra significantly better than the simulations employing Brownian diffusion. The difference between the models is particularly pronounced in the reproduction of the high-field region of the spectra where the random jump model produces flattened lineshapes, in accord with the observed spectra. This conclusion is supported by the dependence of the lineshapes of

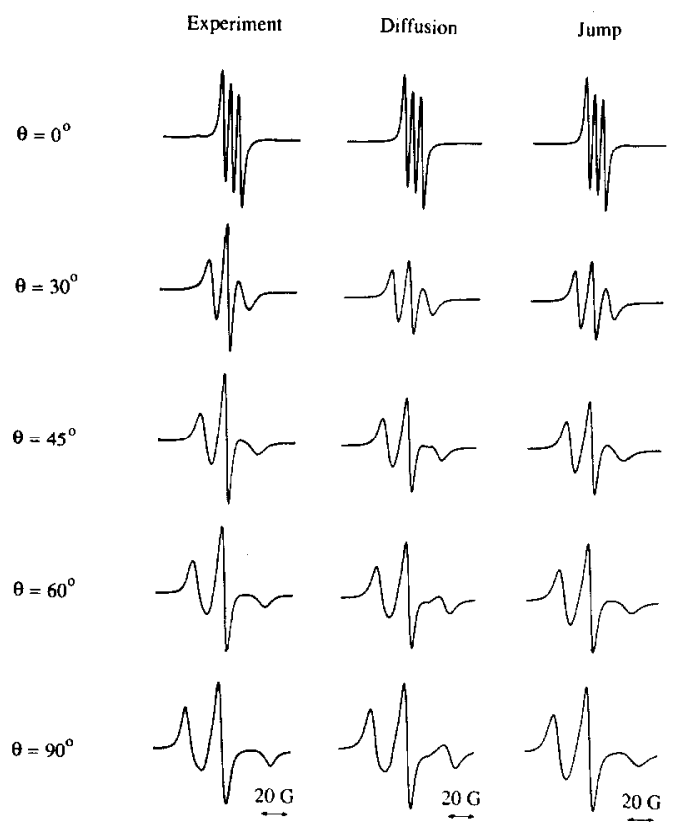

Fig. 8. Dependence of the EPR spectrum of ${ }^{14} \mathrm{~N}-\mathrm{CSL}$ on the angle $\theta$ between the director $\boldsymbol{n}$ and the magnetic field $\boldsymbol{H}_{0}$. Experimental spectra were recorded at $T=18^{\circ} \mathrm{C}$. Calculations were carried out with the parameters given in table 1 (except for an isotropic Gaussian broadening with $\sigma=0.8 \mathrm{G}$ ) and table 2 .
${ }^{14} \mathrm{~N}-\mathrm{CSL}$ on the orientation of the director relative to the magnetic field $H_{0}$. Simulations of the lineshapes for various angles $\theta$ are shown in fig. 8 . Again it is clear that the jump model provides a better description of the motion process. This conclusion contrasts with our earlier study [9] of the same system at $\mathrm{Q}$ band $\left(H_{0} \approx 12.5 \mathrm{kG}\right)$, where neither model provided a satisfactory description of the experimental spectra. The discrepancy may be due to misalignment in the very small samples used in the Q-band experiments. Moreover, we note that the differences in the Q-band lineshapes produced by the models were not so obvious as the ones reported here.

The results summarized in table 2, however, indicate that the simulations of the ${ }^{14} \mathrm{~N}-\mathrm{CSL}$ spectra systematically require higher rates of motion than those needed for simulation of the ${ }^{15} \mathrm{~N}-\mathrm{CSL}$ spectra. The effect appears to be enhanced on lowering the temperature. This finding may be indicative of the limited validity of the planar rotor approximation. With this respect, however, we refer to the simulations of Meirovitch [26], pertaining to ${ }^{14} \mathrm{~N}$-CSL in thiourea cyclohexane single crystals. The spin labels are assumed to undergo small-step Brownian diffusion in the presence of a strong orienting potential (yielding $\left\langle\mathscr{D}_{00}^{2}(\Omega)\right\rangle=0.89$ ). The trends in our spectra, which we interpret in favour of a rotational jump model, are unlike the features of the spectra of Meirovitch. This leads us to believe that our conclusion in favour of a jump model is no artefact of the assumed perfect ordering.

\section{Acknowledgement}

The authors wish to thank Dr. S.D. Venkataramu for generously providing the ${ }^{15} \mathrm{~N}$-CSL spin label employed in these studies. Part of this work was performed while AJD was a visiting scientist at Vanderbilt University. The NATO grant, awarded by the Nederlandse Organisatie voor Zuiver-Wetenschappelijk Onderzoek (ZWO), which enabled this visit, is gratefully acknowledged. This work was also supported by a grant from the National Institute of Health, HL34737, to AHB. 


\section{Appendix}

The factors $F_{p}^{\prime}, A_{p}$ and $A_{p}^{\prime}(p=0, \pm 2)$ appearing in the spin Hamiltonian $\mathscr{H}(\phi)$, as given by eq. (13), are related to the spherical tensor components of $\mathbf{g}$ and $\mathbf{A}$ according to

$F_{p}^{\prime}=\left(\frac{2}{3}\right)^{1 / 2} H_{0} g^{(2, p)}$,

$A_{p}=-\frac{1}{2}\left|\gamma_{\mathrm{e}}\right| A^{(2, p)}$,

$A_{p}^{\prime}=\left(\frac{2}{3}\right)^{1 / 2}\left|\gamma_{\mathrm{e}}\right| A^{(2 . p)}$,

where the following spherical tensor components are employed [14]:

$$
\begin{aligned}
& T^{(2,0)}=\left(\frac{2}{3}\right)^{1 / 2}\left[T_{z z}-\frac{1}{2}\left(T_{x x}+T_{y y}\right)\right], \\
& T^{(2,2)}=T^{(2,-2)}=\frac{1}{2}\left(T_{x x}-T_{y y}\right),
\end{aligned}
$$

while $T$ should be substituted by $g$ or $A$. The absence of off-diagonal elements of $\mathbf{g}$ and $\mathbf{A}$ is a consequence of the fact that these tensors share the same principal axis system. Furthermore, the notation $T_{i} \equiv T_{i i}(i=x, y$ or $z)$ is used throughout the text.

\section{References}

[1] L.J. Berliner, ed., Spin labeling (Academic Press, New York, 1976).

[2] M.A. Hemminga and H.J.C. Berendsen, J. Magn. Res. 8 (1972) 133.

[3] M.A. Hemminga, Chem. Phys. 6 (1977) 87.

[4] M.A. Hemminga, J. Magn. Res. 25 (1977) 25.

[5] C. Mailer, C.P.S. Taylor, S. Schreier-Mucillo and I.C.P. Smith, Arch. Biochem. Biophys. 163 (1974) 671.

[6] Y. Shimoyama, L.E. Göran Eriksson and A. Ehrenberg, Biochim. Biophys. Acta 508 (1978) 213.

[7] M. Ehrström and A. Ehrenberg, Biochim. Biophys. Acta 735 (1983) 271
[8] E. Meirovitch and J.H. Freed, J. Phys. Chem. 84 (1980) 3281.

[9] P. Koole, A.J. Dammers and Y.K. Levine, Chem. Phys. Lip. 35 (1984) 161.

[10] P. Koole, C. Dijkema, G. Casteleijn and M.A. Hemminga, Chem. Phys. Letters 79 (1981) 360.

[11] M. Delmelle, K.W. Butler and I.C.P. Smith, Biochemistry 19 (1980) 698.

[12] J.F.W. Keana, S.B. Keana and D. Beetham, I. Am. Chem. Soc. 89 (1967) 3055.

[13] S.D. Venkataramu. D.E. Pearson, A.H. Beth, K. Balasubramanian, C.R. Park and J.H. Park, J. Label. Compounds Radiopharm. 20 (1983) 433.

[14] M.E. Rose, Elementary theory of angular momentum (Wiley, New York, 1957).

[15] E. Meirovitch, D. Igner, E. Igner, G. Moro and J.H. Freed, J. Chem. Phys. 77 (1982) 3915, and references therein.

[16] J.H. Freed, in: Spin labeling, ed. L.J. Berliner (Academic Press, New York, 1976) ch. 3.

[17] C.F. Polnaszek, G.V. Bruno and J.H. Freed, J. Chem. Phys. 58 (1973) 3189.

[18] G. Moro and J.H. Freed, J. Chem. Phys. 74 (1981) 3757.

[19] A.J. Dammers, Y.K. Levine and J.A. Tjon, Chem. Phys. Letters 88 (1982) 198;

A.J. Dammers, Ph.D. Thesis, University of Utrecht, The Netherlands (1985);

A.J. Dammers, Y.K. Levine and J.A. Tjon, J. Stat. Phys., to be published.

[20] M. Giordano, P. Grigolini, D. Leporini and P. Marin, Phys. Rev. A 28 (1983) 2474.

[21] A.J. Dammers, Y.K. Levine and J.A. Tjon, J. Chem. Phys., to be published.

[22] P.L. Nordio, G. Rigatti and U. Segre, J. Chem. Phys. 56 (1972) 2117

[23] A.E. Stillman and J.H. Freed, J. Chem. Phys. 72 (1980) 550.

[24] L.J. Libertini and O.H. Griffith, J. Chem. Phys. 53 (1970) 1359 ;

G.B. Birell, S.P. Van and O.H. Griffith, J. Am. Chem. Soc. 95 (1973) 2451.

[25] D. Marsh and I.C.P. Smith, Biochim. Biophys. Acta 298 (1973) 133.

[26] E. Meirovitch, J. Phys. Chem. 87 (1983) 3310.

[27] G.R. Luckhurst, M. Setaka and R.N. Yeates, J. Magn. Res. 42 (1981) 351. 